\title{
Mortalidade Cardiovascular Associada ao Rastreamento Mamográfico
}

doi: https://doi.org/10.32635/2176-9745.RBC.2019v65n3.335

\author{
Cardiovascular Mortality Associated with Mammographic Screening \\ Mortalidad Cardiovascular Asociada al Cribado Mamográfico
}

\author{
Arn Migowski'; Paulo Nadanovsky ${ }^{2}$; Cid Manso de Mello Vianna ${ }^{3}$
}

Resumo

Introduçáo: Os danos cardiovasculares do rastreamento mamográfico não têm sido objeto de estudo ou preocupaçóes, inclusive na área de cardio-oncologia. O resultado é uma importante lacuna na literatura a despeito de evidências da grande magnitude do sobrediagnóstico e do sobretratamento no rastreamento e sua ligação com aumento da mortalidade cardiovascular. Objetivo: Apresentar e discutir as principais evidências a respeito das causas de aumento de mortalidade cardiovascular associadas ao rastreamento. Método: Foram realizadas buscas sistemáticas na literatura, por meio de quatro estratégias de busca em duas bases de dados (MEDLINE e LILACS), para identificar as causas de aumento de mortalidade cardiovascular potencialmente associadas ao sobrediagnóstico e ao sobretratamento. Para cada uma das estratégias de busca, os resultados tiveram seu nível de evidência atribuídos de acordo com a classificação do Oxford Centre for Evidence-Based Medicine. Resultados: Dois grandes grupos de causas de aumento da mortalidade cardiovascular foram identificados: o primeiro ligado diretamente ao diagnóstico de câncer de mama; e o segundo ao tratamento do câncer de mama, incluindo cirurgia e radioterapia adjuvante. O aumento de mortalidade cardiovascular incluiu diversos subgrupos de causas, tais como infarto agudo do miocárdio, tromboembolismo pulmonar, insuficiência cardíaca, arritmias, doença orovalvar e acidente vascular encefálico. Conclusáo: Existem evidências consistentes sobre mortalidade cardiovascular associada ao diagnóstico e ao tratamento do câncer de mama em situaçóes clinicamente compatíveis com o rastreamento. É provável também que essa seja uma das causas mais importantes da mortalidade relacionada ao rastreamento, em especial aquelas associadas ao sobretratamento com radioterapia adjuvante.

Palavras-chave: Neoplasias da Mama/diagnóstico; Doenças Cardiovasculares/mortalidade; Programas de Rastreamento; Mamografia; Radioterapia Adjuvante.

\begin{abstract}
Introduction: Cardiovascular harms of mammographic screening have not been the subject of study or concern, including in the cardio-oncology area of. The result is an important gap in literature despite the evidence of great magnitude of overdiagnosis and overtreatment in screening and its association with increased cardiovascular mortality. Objective: Present and discuss the main evidence regarding the causes of increased cardiovascular mortality associated with screening. Method: Systematic searches were performed in the literature through four search strategies in two databases (MEDLINE and LILACS), to identify the causes of increased cardiovascular mortality potentially associated with overdiagnosis and overtreatment. For each one of the search strategies, it was used the classification of the Oxford Centre for Evidence-Based Medicine to assign the level of evidence of the results. Results: Two major groups of causes of increased cardiovascular mortality were identified: the first linked directly to the diagnosis of breast cancer; and the second to the treatment of breast cancer, including surgery and adjuvant radiotherapy. The increase of cardiovascular mortality included several subgroups of causes such as acute myocardial infarction, pulmonary thromboembolism, heart failure, arrhythmias, heart valve disease and stroke. Conclusion: There are consistent evidence about cardiovascular mortality associated with breast cancer diagnosis and treatment in conditions clinically compatible with screening. It is also likely to be one of the most important causes of mortality related to screening, especially those associated with overtreatment with adjuvant radiotherapy.

Key words: Breast Neoplasms/diagnosis; Cardiovascular Diseases/mortality; Mass Screening; Mammography; Radiotherapy, Adjuvant.
\end{abstract}

\section{Resumen}

Introducción: El daño cardiovascular causado por el cribado mamográfico no ha sido objeto de estudio ni de preocupación, incluso en el área de la cardiooncología. El resultado es una brecha importante en la literatura a pesar de la evidencia de la gran magnitud del sobrediagnóstico y el sobretratamiento en cribado. y su asociación con el aumento de la mortalidad cardiovascular. Objetivo: Presentar y discutir los principales pruebas en las causas del aumento de la mortalidad cardiovascular asociados con la tamización. Método: Se realizaron búsquedas sistemáticas en la literatura a través de cuatro estrategias de búsqueda en dos bases de datos (MEDLINE y LILACS), para identificar las causas del aumento de la mortalidad cardiovascular potencialmente asociadas con el sobrediagnóstico y el sobretratamiento. Para cada una de las estrategias de búsqueda, a los resultados se les asignó su nivel de evidencia de acuerdo con la clasificación del Oxford Centre for Evidence-Based Medicine. Resultados: Se identificaron dos grupos principales de causas de aumento de la mortalidad cardiovascular: el primero relacionado directamente con el diagnóstico de cáncer de mama; y el segundo para el tratamiento del cáncer de mama, incluida la cirugía y la radioterapia adyuvante. El aumento de la mortalidad cardiovascular incluyó varios subgrupos de causas como infarto agudo de miocardio, tromboembolismo pulmonar, insuficiencia cardíaca, arritmias, enfermedad orovalvar y accidente cerebrovascular. Conclusión: Existe evidencia consistente de mortalidad cardiovascular asociada con el diagnóstico y tratamiento del cáncer de mama en condiciones clínicamente compatibles con la tamización. También es probable que sea una de las causas más importantes de mortalidad relacionada con la tamización, especialmente aquellas asociadas con el sobretratamiento con radioterapia adyuvante.

Palabras clave: Neoplasias de la Mama/diagnóstico; Enfermedades Cardiovasculares/mortalidad; Tamizaje Masivo; Mamografía; Radioterapia Ayuvante.

\footnotetext{
${ }^{1}$ Instituto Nacional de Cardiologia (INC). Instituto Nacional de Câncer José Alencar Gomes da Silva (INCA). Orcid iD: https://orcid.org/0000-0002-4861-2319 ${ }^{2}$ Instituto de Medicina Social/Universidade do Estado do Rio de Janeiro (IMS/Uerj). Escola Nacional de Saúde Pública. Fundação Oswaldo Cruz (Ensp/Fiocruz). Orcid iD: https://orcid.org/0000-0003-3345-9873

${ }^{3}$ IMS/Uerj. Orcid iD: https://orcid.org/0000-0003-0252-1144

Endereço para correspondência: Arn Migowski. Rua das Laranjeiras, 374 - 5ªndar - Laranjeiras. Rio de Janeiro (RJ), Brasil. CEP $22240-002$.

E-mail: arnmigowski@yahoo.com.br
} 


\section{INTRODUÇÃO}

É sabido que o rastreamento do câncer de mama com mamografia aumenta o diagnóstico de casos de câncer de mama que nunca iriam se manifestar clinicamente (sobrediangóstico) ${ }^{1-3}$ e que esse aumento de casos diagnosticados também resulta em tratamento oncológico desnecessário (sobretratamento) ${ }^{4}$. Esse aumento de tratamento é contraintuitivo na medida em que se esperava que, com o rastreamento, haveria reduçáo da morbidade. Porém, o fato é que os casos de sobrediagnóstico nunca evoluiriam para estadiamentos mais avançados, mesmo sem tratamento. A existência do sobretramento pelo rastreamento mamográfico já está comprovada para a radioterapia adjuvante e cirurgia (conservadora e mastectomia) ${ }^{4}$.

Contudo, os danos associados ao rastreamento mamográfico raramente são incluídos em estudos primários, revisôes ou diretrizes clínicas. Mesmo os riscos mais comuns do rastreamento como resultados falso-positivos e sobrediagnóstico, geralmente, não são considerados ${ }^{1}$. Além disso, é preciso entender que o sobrediagnóstico e o sobretratamento são desfechos intermediários, e muito pouca atenção tem sido dada ao estudo dos danos à saúde produzidos por essas duas condiçōes ${ }^{2}$. Particularmente, as causas de aumento de mortalidade cardiovascular com o rastreamento mamográfico não têm sido objeto de estudo ou preocupação, inclusive na área de cardio-oncologia. $\mathrm{O}$ resultado é uma importante lacuna na literatura científica internacional a despeito das evidências sobre a importante magnitude do sobrediagnóstico ${ }^{3}$ e do sobretratamento . $^{4}$ Se os danos cardiovasculares do sobretratamento fossem considerados, é possível - segundo algumas estimativas - que a mortalidade associada ao rastreamento superasse até mesmo o benefício na redução da mortalidade por câncer de mama, em virtude do sobrediagnóstico e do sobretratamento ${ }^{5}$, demonstrando a importância de um aprofundamento no tema.

\section{OBJETIVO}

A presente revisão tem por objetivo apresentar e discutir as principais evidências disponíveis a respeito das causas de aumento de mortalidade cardiovascular associadas ao diagnóstico e ao tratamento do câncer de mama em situaçôes clinicamente compatíveis com o sobrediagnóstico e o sobretratamento derivados do rastreamento mamográfico.

\section{MÉTODO}

A partir de uma revisão sistemática da literatura sobre riscos e benefícios do rastreamento mamográfico previamente realizada pelos autores, cujos métodos estấo descritos com detalhes em outras publicaçôes ${ }^{1,2}$, foram definidas quais situações envolvendo o diagnóstico e o tratamento do câncer de mama poderiam ser consideradas como consequência do rastreamento mamográfico. Esta revisão sistemática evidenciou ainda que os potenciais danos cardiovasculares associados ao sobrediagnóstico e ao sobretratamento não foram estudados diretamente em ensaios clínicos de rastreamento mamográfico ${ }^{2}$.

Dessa forma, para o presente artigo, foram empreendidas novas buscas sistemáticas na literatura, com o intuito de produzir uma revisáo sobre as causas de aumento de mortalidade cardiovascular potencialmente associadas ao sobrediagnóstico ou ao tratamento desnecessário consequentes ao rastreamento mamográfico. Como o sobrediagnóstico e o sobretratamento são contrafactuais, não é possível estudar diretamente esses casos, sendo necessário identificar quais situaçóes clínicas seriam mais compatíveis com eles. Isso ocorre, pois, no nível individual, não se pode determinar com precisão quem de fato foi sobrediagnosticado ou tratado desnecessariamente. Nessas situaçóes, o aumento de mortalidade cardiovascular é mais relevante, pois não há uma contrapartida dos benefícios do rastreamento mamográfico na redução da mortalidade por câncer de mama, já que, por definição, trata-se de diagnóstico e tratamento de um câncer que não evoluiria clinicamente, mesmo se não detectado ${ }^{1}$. A longa sobrevida dos casos de sobrediagnóstico também os torna mais suscetíveis aos danos de longo prazo da terapia oncológica.

As situaçôes consideradas clinicamente compatíveis com o rastreamento foram as seguintes: diagnóstico de carcinoma ductal in situ (CDIS) ou câncer de mama invasivo em estádio inicial/localizado; cirurgia (incluindo a conservadora e a mastectomia) ou radioterapia adjuvante para tratamento de CDIS ou câncer de mama invasivo em estádio inicial/localizado. Por falta de evidências conclusivas na literatura ${ }^{2,4}$ a respeito de sua associação com o rastreamento mamográfico, as possibilidades de sobretratamento com hormonioterapia, quimioterapia e trastuzumabe não foram incluídas nas buscas ou nos critérios de elegibilidade para seleção de artigos na presente revisão, tendo sido abordados apenas na discussão do presente artigo para fins de completude da abordagem do tema. Importante ressaltar que como danos cardiovasculares do sobrediagnóstico do câncer de mama, não foram abordadas as complicaçóes advindas da doença câncer de mama que, nos casos de sobrediagnóstico, nem sequer existe clinicamente, mas sim dos impactos iatrogênicos da investigação diagnóstica e do próprio diagnóstico per se.

A pergunta de pesquisa foi definida pelo acróstico PICO, sendo a situação clínica estudada o diagnóstico e 
o tratamento de câncer de mama localizado (in situ ou invasivo) compatíveis com casos identificados por meio de rastreamento mamográfico (população/intervenção), comparados com a ausência de rastreamento (e consequente ausência do diagnóstico e do tratamento), tendo como desfecho a mortalidade por causas cardiovasculares. Dessa forma, buscou-se identificar as causas desse aumento de mortalidade cardiovascular. A busca foi realizada nas bases MEDLINE e LILACS. Para cada causa de óbito cardiovascular identificada, foram realizadas verificaçôes das listas de referência dos artigos recuperados na MEDLINE nas buscas específicas para aquela causa. Na LILACS, a estratégia de busca utilizada foi: mama [Palavras do título] and câncer [Palavras do título] and cardiovascular [Palavras do resumo]. Na MEDLINE, as buscas envolveram as três estratégias de busca descritas a seguir:

\section{Busca sobre danos cardiovasculares do sobretratamento com cirurgia:}

(((breast[tiab] OR mamar*[tiab]) AND (tumor[tiab] OR tumors[tiab] OR cancer[tiab] OR neoplasm*[tiab] OR carcinoma[tiab] OR "Breast Neoplasms"[Majr] OR "Breast Neoplasms"[Mh]) AND ("breast cancer surgery” OR mastectomy OR lumpectomy) AND ("mortality rates" OR "post-surgical complication*" OR "post-surgical mortality" OR "cardiovascular death" OR "cardiovascular mortality" OR "In-hospital case-fatality rates" OR “cardiac death”)) AND ( "1990/01/01”[PDat] : “2019/08/31”[PDat] ))

\section{Busca sobre danos cardiovasculares do sobretratamento com radioterapia:}

(()((systematic review[ti] OR meta-analysis[pt] OR meta-analysis[ti] OR systematic literature review[ti] OR (systematic review[tiab] AND review[pt]) OR consensus development conference[pt] OR practice guideline[pt] OR cochrane database syst rev[ta] OR acp journal club[ta] OR health technol assess[ta] OR evid rep technol assess summ[ta] OR drug class reviews[ti]) OR (clinical guideline [tw] AND management[tw]) OR ((evidence based[ti] OR evidence-based medicine[mh] OR best practice* [ti] OR evidence synthesis[tiab]) AND (review[pt] OR diseases category[mh] OR behavior and behavior mechanisms[mh] OR therapeutics[mh] OR evaluation studies[pt] OR validation studies[pt] OR guideline[pt] OR pmcbook)) OR (systematic[tw] OR systematically[tw] OR critical[tiab] OR (study selection [tw]) OR (predetermined[tw] OR inclusion[tw] AND criteri*[tw]) OR exclusion criteri*[tw] OR main outcome measures[tw] OR standard of care[tw] OR standards of care[tw]) AND (survey[tiab] OR surveys[tiab] OR overview*[tw] OR review[tiab] OR reviews[tiab] OR search*[tw] OR handsearch[tw] OR analysis[tiab] OR critique[tiab] OR appraisal[tw] OR (reduction[tw] AND (risk[mh] OR risk[tw]) AND (death OR recurrence))) AND (literature[tiab] OR articles[tiab] OR publications[tiab] OR publication[tiab] OR bibliography[tiab] OR bibliographies[tiab] OR published[tiab] OR unpublished[tw] OR citation[tw] OR citations[tw] OR database[tiab] OR internet[tiab] OR textbooks[tiab] OR references[tw] OR scales[tw] OR papers[tw] OR datasets[tw] OR trials[tiab] OR meta-analy*[tw] OR (clinical[tiab] AND studies[tiab]) OR treatment outcome[mh] OR treatment outcome[tw] OR pmcbook)) AND ((clinical[tiab] AND trial[tiab]) OR clinical trial* $\left.{ }^{*} \mathrm{mh}\right]$ OR clinical trial[pt] OR random*[tiab] OR random*[tw] OR random allocation[mh] OR "randomized controlled trial" [pt] OR "controlled clinical trial”[pt]) AND (breast[tiab] OR mamar*[tiab]) AND (tumor[tiab] OR tumors[tiab] OR cancer[tiab] OR neoplasm*[tiab] OR carcinoma[tiab] OR "Breast Neoplasms"[Majr] OR "Breast Neoplasms"[Mh] OR DCIS OR "Ductal Carcinoma in situ") AND (cardiovascular[tiab] OR heart[tiab] OR cardiac[tiab]) AND (Radiotherapy OR "Radiation Therapy")))) AND (“1990/01/01”[PDat] : “2019/08/31”[PDat] ))

\section{Busca sobre danos cardiovasculares do sobrediagnóstico:}

("cardiovascular death" OR "cardiovascular mortality" OR "cardiac death") AND ("cancer diagnosis" OR overdiagnosis) AND ("1990/01/01”[PDat] : “2019/05/31"[PDat])

Não foi feita restrição de idiomas em nenhuma das estratégias de busca realizadas. Por ser um tema mais estudado na literatura, na seleção das referências sobre os danos cardiovasculares do tratamento com radioterapia adjuvante, foi exigido também que o desenho de estudo fosse ensaio clínico randomizado ou revisão sistemática de ensaios clínicos randomizados.

A classificação do Oxford Centre for Evidence-Based Medicine foi utilizada como parâmetro para se identificar o maior nível de evidência disponível ${ }^{6}$. Essa classificação possui cinco níveis, sendo o nível 1 o de melhor evidência e o nível 5 o de pior e tem a vantagem de possuir uma classificação específica para perguntas de pesquisa sobre danos à saúde ${ }^{6}$. Optou-se por focar a revisão na mortalidade cardiovascular (total ou por subgrupos) por ser um desfecho duro, de grande relevância de saúde pública e cuja aferição é mais objetiva.

\section{RESULTADOS}

Foram encontradas evidências a respeito de dois grandes grupos de causas de aumento da mortalidade 
cardiovascular: um ligado diretamente ao próprio diagnóstico de câncer de mama e outro, ao tratamento do câncer de mama. Esta última categoria foi subdividida pelas diversas modalidades terapêuticas utilizadas no tratamento oncológico de casos de câncer de mama localizado, incluindo cirurgia e radioterapia adjuvante.

No primeiro grupo, estão as mortes cardiovasculares associadas diretamente ao diagnóstico do câncer de mama. Nessa busca, foram recuperados 52 artigos na MEDLINE, sendo três deles mantidos para a leitura na íntegra, sendo dois deles selecionados ao final do processo ${ }^{7,8}$. Dos estudos selecionados para leitura na íntegra, um foi excluído por não apresentar os danos associados diretamente ao diagnóstico/sobrediagnóstico do câncer de mama ${ }^{9}$. Ambos os estudos selecionados são de coorte de base populacional e foram classificados como nível 3 de evidência. Não foram identificadas referências a respeito de mortes relacionadas às biópsias de mama.

Para a mortalidade em virtude do tratamento com cirurgia mamária, tanto por mortalidade cirúrgica quanto por complicaçóes de longo prazo, foram recuperados 83 artigos na MEDLINE, sendo quatro mantidos para leitura na íntegra e apenas um selecionado de acordo com os critérios de elegibilidade ${ }^{10}$. Dos estudos selecionados para leitura na íntegra, um foi excluído por não apresentar resultados de complicaçôes cardiovasculares do tratamento cirúrgico $^{11}$. O estudo selecionado refere-se a uma série de casos, tendo sido classificado como nível 4 de evidência.

$\mathrm{Na}$ mortalidade cardiovascular associada à radioterapia adjuvante, foram recuperados 43 artigos na MEDLINE, sendo quatro mantidos para leitura na íntegra e apenas um deles selecionado de acordo com os critérios de elegibilidade $^{12}$. Dos estudos mantidos para leitura na íntegra, um foi excluído por ser uma revisão sistemática que incluiu apenas estudos observacionais ${ }^{13}$, e dois foram excluídos por não especificarem o estadiamento dos casos de câncer de mama analisados ${ }^{14,15}$. O estudo selecionado foi uma metanálise de ensaios clínicos randomizados de tratamento do câncer de mama localizado, tendo sido classificado como nível 1 de evidência.

As buscas na LILACS retornaram 36 referências, nenhuma das quais foi selecionada de acordo com os critérios de elegibilidade definidos.

\section{DISCUSSÃO}

Em nosso melhor conhecimento, a presente revisão é a primeira na literatura mundial a abordar todas as possíveis causas de aumento de mortalidade cardiovascular associadas ao rastreamento do câncer de mama. É conhecido que mulheres sobreviventes de câncer de mama possuem maior mortalidade cardiovascular do que a populaçáo em geral ${ }^{16}$. Porém, esses estudos tratam a sobrevivência de câncer sem considerar que boa parte desses casos na verdade se refere a sobrediagnóstico resultante do rastreamento mamográfico ${ }^{5}$. Além disso, os estudos sobre o aumento da mortalidade cardiovascular após o diagnóstico de câncer de mama são considerados limitados e nenhum deles focou especificamente em situaçôes clínicas associadas ao rastreamento do câncer de mama ou buscou de forma ampla as várias causas desse aumento ${ }^{16}$. A seguir, serão discutidos separadamente os resultados para cada uma das causas de aumento de mortalidade cardiovascular potencialmente associadas ao rastreamento mamográfico.

\section{MORTALIDADE CARDIOVASCULAR LIGADA DIRETAMENTE AO SOBREDIAGNÓSTICO}

As principais evidências de aumento do risco de morte cardiovascular logo após o diagnóstico de câncer de mama vêm de um estudo de coorte envolvendo 74.977 mulheres na Suécia, selecionado na presente revisão ${ }^{7}$. Esse aumento foi estimado de acordo com o tempo após o diagnóstico de câncer de mama, medido em semanas, com o objetivo de separar o efeito do diagnóstico das possíveis consequências do tratamento 7 . Nesse estudo, o risco de morte cardiovascular (CID 10: I00-I99) na primeira semana após o diagnóstico foi maior do que nas semanas subsequentes ${ }^{7}$. O risco relativo (RR) de mortalidade por todas as causas cardiovasculares na primeira semana foi de 1,8 (IC 95\%: 1,2-2,4) no modelo multivariado com risco ajustado para possíveis fatores de confusão como idade, estado civil, nível socioeconômico e escolaridade, permanecendo significativamente elevado por um mês ${ }^{7}$.

Confirmou-se ainda que o risco relativo de morte cardiovascular após diagnóstico foi maior naquelas pacientes que não possuíam doenças psiquiátricas preexistentes, o que também ocorreu para aumento do risco de suicídio após o diagnóstico de câncer de mama ${ }^{7}$. A análise estratificada por grupo de causas cardiovasculares mostrou o aumento estatisticamente significativo de risco de morte cardiovascular em todos os subgrupos cardiovasculares estudados em até quatro semanas após o diagnóstico de câncer. Esses subgrupos foram infarto agudo do miocárdio, "embolismo ou trombose", acidente vascular encefálico (isquêmico ou hemorrágico) e "outras doenças do coração" .

Dos danos cardiovasculares identificados na presente revisão, o aumento de mortalidade cardiovascular logo em seguida ao diagnóstico de câncer de mama é sem dúvidas o menos conhecido. A explicação mais aceita para esse fenômeno seria de que o diagnóstico de câncer, por seu estigma de doença letal e causadora de sofrimento, funcionaria como um "evento vital estressante", o que 
está associado a diversos desfechos cardiovasculares desfavoráveis ${ }^{8}$. Resultados em modelo animal têm comprovado que o estresse psicológico agudo está relacionado ao desencadeamento de isquemia miocárdica, arritmogênese, agregação plaquetária, disfunção endotelial, aumento da viscosidade sanguínea por hemoconcentração, aumento da pressão arterial sistêmica e vasoconstrição ${ }^{17}$.

Diversas pesquisas têm associado estresse psicológico agudo grave com o desencadeamento de infarto agudo do miocárdio e morte súbita cardíaca. Entre os desencadeantes descritos nesses estudos, estão os eventos estressores vitais, tais como o diagnóstico de câncer, ocorrência de desastres naturais, ataques militares e falecimento de familiares, além de outros relacionados à vida financeira e ocupacional ${ }^{17-21}$. Por exemplo, a Women's Health Initiative - uma coorte de mulheres na pós-menopausa - identificou associação estatisticamente significativa entre eventos vitais estressantes com aumento de incidência de doença arterial coronariana e acidente vascular encefálico, independente de fatores sociodemográficos e de sintomas depressivos ${ }^{22}$.

Outro quadro cardiovascular de alta letalidade, a dissecção aórtica aguda, também pode ser desencadeado por estresse psicológico. Em uma série de 175 casos consecutivos em um hospital de referência nos Estados Unidos da América (EUA), o início do quadro de dissecção aórtica aguda esteve relacionado a estresse psicológico em $40 \%$ dos casos, incluindo o diagnóstico de câncer ${ }^{14}$. A provável explicação fisiopatológica para essa associação seria o aumento agudo de pressão arterial sistêmica com o estresse, que excederia o limite de tração do tecido aórtico ${ }^{23}$.

Outra possível causa seria a miocardiopatia de Takotsubo, também chamada de miocardiopatia induzida por estresse, de insuficiência cardíaca aguda relativamente pouco conhecida. Ela mimetiza o diagnóstico de infarto agudo do miocárdio, sendo o diagnóstico final de $2 \%$ dos casos inicialmente atendidos com síndrome coronariana aguda ${ }^{24}$. A miocardiopatia de Takotsubo apresenta episódios de estresse psicológico como fator precipitante em cerca de 30\% dos casos, sendo mais comum em mulheres e, assim como o câncer de mama, afeta predominantemente mulheres na pós-menopausa ${ }^{24}$.

Portanto, conclui-se, de acordo com a literatura, que há plausibilidade biológica para a associação de diversos diagnósticos cardiovasculares com o aumento de mortalidade cardiovascular observado logo após o diagnóstico do câncer de mama, tais como infarto agudo do miocárdio, acidente vascular encefálico, morte súbita cardíaca, tromboembolismo, dissecção aórtica aguda e miocardiopatia de Takotsubo. Outra hipótese explicativa alternativa para a mortalidade cardiovascular logo em seguida ao diagnóstico de câncer seria a possibilidade de complicaçôes decorrentes de biópsias, não tendo, contudo, suporte na literatura ${ }^{25,26}$.

\section{MORTALIDADE CARDIOVASCULAR LIGADA AO SOBRETRATAMENTO COM CIRURGIA}

A mortalidade e até mesmo a incidência de desfechos cardiovasculares graves em razáo da mastectomia e das cirurgias conservadoras parecem ser baixas de acordo com a literatura, embora seja necessário fazer a ressalva de que esse é um tema relativamente pouco estudado. Em uma grande série multicêntrica de casos nos EUA selecionada na presente revisão, a ocorrência de acidente vascular encefálico no pós-operatório foi de $0,1 \%$ tanto no grupo submetido à mastectomia quanto no grupo submetido às cirurgias conservadoras, não sendo, contudo, observado nenhum óbito no último grupo ${ }^{10}$. Nesse mesmo estudo, a incidência de infarto agudo do miocárdio em até 30 dias após a mastectomia foi de $0,06 \%$, mesmo percentual observado para morte súbita de origem cardíaca ${ }^{10}$. A miocardiopatia de Takotsubo também está descrita como complicação rara no pós-operatório de cirurgia conservadora para câncer de $\operatorname{mama}^{27}$.

\section{MORTALIDADE CARDIOVASCULAR LIGADA AO SOBRETRATAMENTO COM RADIOTERAPIA}

A radioterapia para o câncer de mama também aumenta o risco de diversas doenças cardiovasculares, como a doença cardíaca isquêmica, cardiomiopatia, pericardite e doença orovalvar actínica ${ }^{28,12}$. Esse risco cresce mais acentuadamente quando a mama esquerda é irradiada ${ }^{29}$. Por isso, muitas vezes, mulheres com irradiação para câncer na mama direita são utilizadas como grupo controle de estudos que avaliam os riscos cardíacos da radioterapia. Uma recente revisão sistemática de estudos observacionais evidenciou um aumento estatisticamente significativo de $23 \%$ na mortalidade cardiovascular nesses casos, quando comparados a casos em que a mama direita foi irradiada ${ }^{13}$. Em outra metanálise, que reuniu dados de ensaios clínicos randomizados e estudos observacionais, pacientes tratadas com radioterapia tiveram risco 38\% maior de mortalidade cardíaca (IC 95\%: 1,18-1,62) comparadas às pacientes náo submetidas à radioterapia, correspondendo a um aumento absoluto de 125,5 mortes cardíacas por 100 mil pessoas-ano ${ }^{30}$.

Em outra metanálise de ensaios clínicos, os riscos relativos de morte por doença arterial coronariana, insuficiência cardíaca não isquêmica, arritmia, tromboembolismo pulmonar (TEP) e doença orovalvar no grupo tratado com radioterapia adjuvante foram respectivamente $1,31,1,94,2,14,2,10$ e 1,97 , todos eles com significância estatística ${ }^{21}$. O risco de eventos coronarianos graves (definidos como infarto agudo do 
miocárdio, revascularização coronariana ou óbito por doença isquêmica do coração) aumenta linearmente com a dose de radiaçáo e náo parece haver um limite inferior de segurança para o qual náo existiria aumento de risco ${ }^{12,31}$. Há indícios de que o aumento de risco de complicaçóes cardiovasculares seja maior nas mulheres tratadas antes dos 50 anos, tanto para doença cardíaca isquêmica, quanto para orovalvar e insuficiência cardíaca ${ }^{29}$. Contudo, os dados de metanálise de ensaios clínicos de radioterapia adjuvante não são conclusivos sobre essa questão ${ }^{12}$.

A relaçáo causal entre a radioterapia e o aumento de mortes por TEP não é bem entendida. O TEP é uma causa conhecida de mortalidade em pacientes com câncer em geral, sendo sua incidência quatro a seis vezes maior nesses pacientes do que na populaçáo em geral ${ }^{132}$. Além do aumento de risco de trombose pelo próprio câncer, a terapia oncológica aumenta ainda mais o risco de TEP. Isso está bem estabelecido para cirurgia, quimioterapia e hormonioterapia, sendo ainda pouco conhecido no caso da radioterapia ${ }^{32}$. Um metanálise de ensaios clínicos de radioterapia adjuvante para câncer de mama mostrou aumento de mortalidade por TEP nessas pacientes (RR de 1,94$)^{33}$. O possível mecanismo fisiopatológico seria destruição celular e promoção de processos inflamatórios pró-trombóticos pela radiação ionizante ${ }^{32}$.

Com a evoluçẫo das técnicas de radioterapia nas últimas décadas, houve uma queda da dose total no coração (6,3 para 4,4 Gy) e no pulmáo ( 9,6 para 5,7 Gy) quando as doses médias na década atual são comparadas às doses nos ensaios clínicos clássicos de radioterapia adjuvante para câncer de mama ${ }^{12}$. Os efeitos dessa redução de dose na diminuição de riscos de longo prazo ainda não são bem conhecidos $^{12}$. A irradiaçáo da artéria descendente anterior esquerda - em função de sua localização anatômica vulnerável e de sua importância na irrigação das paredes anterior, septal e lateral do ventrículo esquerdo - é um dos principais problemas que ainda precisa ser superado ${ }^{34}$.

A nova técnica de inspiração profunda com suspensão da respiração pode diminuir a dose de radiação no coração, tanto na radioterapia de intensidade modulada quanto na arcoterapia volumétrica modulada ${ }^{35}$. Outras técnicas alternativas de radioterapia, como a irradiação parcial da mama, poderáo diminuir ainda mais esses riscos no futuro, mas ainda permanecem experimentais ${ }^{36}$.

O intervalo de tempo após o tratamento em que os danos da radioterapia adjuvante começam a surgir e a sua duração ainda não são consenso na literatura. $\mathrm{O}$ modelo proposto por Baum ${ }^{5}$ considerou que o aumento de risco de infarto agudo do miocárdio e de câncer de pulmáo começa logo após a radioterapia e dura por 30 anos $^{5}$. Em uma metanálise de ensaios clínicos de radioterapia adjuvante para câncer de mama, a mortalidade por outras causas, que não câncer de mama (incluindo cardiovasculares, câncer de pulmão e câncer de esôfago), foi estatisticamente maior no grupo tratado com radioterapia nos períodos de cinco a 14 anos após o tratamento e no período de 15 anos ou mais ${ }^{33}$.

Em um estudo caso-controle de base populacional, o incremento de risco começou cinco anos após a radioterapia e continuou até pelo menos 20 anos $^{31}$. Ele sugeriu ainda que mulheres com tumores impalpáveis, descobertos pelo rastreamento, teriam incremento de risco de evento coronariano maior do que aquelas com tumores maiores (RR respectivamente de 20,4 e 6,9), embora a diferença entre os dois grupos não tenha significância estatística ${ }^{31}$.

Em uma metanálise com 45 ensaios clínicos, a mortalidade por doenças cardiovasculares (excluindo doença cerebrovascular e TEP) após a radioterapia adjuvante para câncer de mama parece aumentar logo no primeiro quinquênio após o tratamento ( $R R$ de 1,40$)$, persistindo até mais de 15 anos após a terapia (RR de $1,42)^{12}$. Embora o risco relativo seja semelhante nesses dois casos, o risco absoluto é maior nas mulheres mais velhas, em funçáo do maior risco de base de morte por doença cardiovascular ${ }^{12}$.

Segundo dados do Surveillance, Epidemiology and End Results, a mortalidade por doenças cardiovasculares cresce já nos primeiros cinco anos após o tratamento (RR de 1,19) e continua até mais de 20 anos (RR de 1,90), aumentando com o tempo, ao menos para as coortes tratadas na década de 1970 e início de $1980^{37}$.

Com relação aos danos de longo prazo da radioterapia - especialmente em mais de 15 anos após o tratamento - é provável que haja um efeito de coorte também já que mulheres com mais tempo de seguimento também são aquelas que foram tratadas com técnicas mais antigas e maior dose de radiação. $\mathrm{O}$ aumento de risco de morte por todas as causas, exceto câncer de mama, é observado ainda nas coortes tratadas mais recentemente, embora com intensidade menor do que naquelas tratadas na década de $1970^{12}$. Os efeitos na mortalidade cardiovascular nos primeiros cinco anos (RR de 1,40) são da mesma magnitude dos produzidos em 15 anos ou mais ${ }^{12}$. Isso fala a favor do maior impacto da mortalidade nos primeiros anos após o tratamento, mesmo com o efeito de coorte tendendo a aumentar o risco em grupos com maior tempo de seguimento. Ainda assim, esse é um tema controverso com estudos favoráveis e contrários a esse aumento precoce de risco ${ }^{29,31}$.

Deve-se considerar que a presente discussão se refere aos danos associados à radioterapia adjuvante associada rastreamento mamográfico. Como a terapia conservadora é mais comum nesse contexto do que a mastectomia, é natural que a radioterapia adjuvante se destaque. Também é necessário entender que os cenários aqui 
analisados dizem respeito ao sobretratamento, onde os riscos da radioterapia náo são contrabalançados com seus benefícios na redução da mortalidade por câncer de mama. Considerando casos de câncer de mama detectados por imagem, mas com significância clínica em mulheres com 50 anos ou mais, o benefício absoluto da radioterapia adjuvante após cirurgia conservadora na redução da mortalidade por câncer de mama ficaria em torno de $2 \%$ a $5 \%{ }^{12}$, além de garantir resultados oncológicos semelhantes ao da mastectomia, mas com melhor resultado estético e redução de complicações cirúrgicas.

Como o aumento da mortalidade por doença arterial coronariana e câncer de pulmão são os maiores riscos associados ao sobretratamento com radioterapia, a existência de um risco de base elevado para essas duas doenças pode resultar em um número ainda maior de óbitos. O caso mais importante é o do tabagismo, por aumentar tanto o risco de câncer de pulmão quanto o de doença arterial coronariana, a ponto de os riscos da radioterapia adjuvante superarem seus benefícios em mulheres com alta carga tabágica, mesmo considerando também os casos em que náo houve tratamento desnecessário (sobretratamento) ${ }^{12}$. É também fator de risco para TEP e câncer de esôfago. Para tabagistas com alta carga tabágica, o tratamento com radioterapia adjuvante aumentaria o risco absoluto de incidência de câncer de pulmão em $4,4 \%$ e por morte por doenças cardíacas em $1,2 \%{ }^{12}$. A cessação do tabagismo diminuiria esse risco absoluto e, até mesmo, caso a mesma ocorresse já no momento do início da radioterapia, seria capaz de diminuir esses riscos do tratamento, já que seus efeitos começam principalmente dez anos após a cessação, quando também são mais altos os riscos de mortalidade cardiovascular pela radioterapia adjuvante ${ }^{12}$.

Outra perspectiva de redução do sobretratamento com radioterapia é a avaliada no ensaio clínico PRIME II, que compara a cirurgia conservadora com e sem radioterapia adjuvante em mulheres com câncer de mama de bom prognóstico. Contudo, neste estudo, houve um pequeno benefício na diminuição da recorrência local com a radioterapia, perpetuando a controvérsia sobre qual seria a melhor conduta, tendo em vista os fatores prognósticos disponíveis ${ }^{38}$.

\section{SOBRETRATAMENTO COM HORMONIOTERAPIA}

Nas últimas décadas, a hormonioterapia se consagrou na prática clínica como parte da terapia adjuvante do câncer de mama ${ }^{39}$, a partir de resultados de ensaios clínicos que demonstraram reduçáo de mortalidade com o uso de tamoxifeno adjuvante por cinco anos ${ }^{33}$.

Por seu uso de rotina no tratamento de tumores localizados e, particularmente, naqueles de bom prognóstico por possuírem receptores hormonais, é muito provável que exista sobretratamento com hormonioterapia na prática clínica. Todavia, uma metanálise de ensaios clínicos randomizados de rastreamento mamográfico ${ }^{4}$ não comprovou esse aumento, o que talvez esteja associado à data em que esses ensaios foram feitos. Para a maioria dos ensaios clínicos randomizados, não há informação sobre tratamento com hormonioterapia. Os dois ensaios com informação a respeito mostram percentual de utilização de apenas $17 \%$ e $2 \% 4$.

Assumindo a existência de sobretratamento com hormonioterapia, esta implica na presença de riscos associados ao uso de tamoxifeno, tais como o aumento de incidência de doença tromboembólica e de câncer uterino. Por sua vez, esses riscos seriam contrabalançados pelo pequeno efeito protetor do tamoxifeno na incidência de doença arterial coronariana ${ }^{26}$. Estima-se que a relação entre riscos e benefícios dos inibidores da aromatase também estejam em equilíbrio, não alterando a mortalidade geral $^{26,40}$.

\section{SOBRETRATAMENTO COM QUIMIOTERAPIA}

No protocolo brasileiro de tratamento do câncer de mama, nos casos de câncer invasivo localizado de baixo risco; ou seja, casos com bom prognóstico e mais compatíveis com possível sobrediagnóstico, o clássico esquema quimioterápico (ciclofosfamida, metotrexato e 5-fluorouracil - CMF) ainda é recomendado, ainda que antraciclinas (adriamicina/doxorrubicina) também sejam consideradas como uma opção em casos individualizados ${ }^{41}$. Já para casos de risco intermediário ou alto, geralmente o regime contém antraciclina ${ }^{41}$.

Considerando a intençẫo curativa dessas terapias, o tempo de sobrevida relativamente longo e a possibilidade da existência de sobretratamento, é essencial conhecer a toxicidade dos regimes de quimioterapia utilizados nesse contexto e seus efeitos em médio e longo prazos. O número de óbitos por toxicidade aguda durante o tratamento com CMF em carcinoma ductal invasivo de mama é de três em mil $(0,3 \%)^{26}$. Os esquemas incluindo antraciclinas apresentam maior cardiotoxicidade do que o CMF e aumentam o risco de insuficiência cardíaca em longo prazo $^{29,42}$. Em menos de $1 \%$ dos casos, esses esquemas podem gerar complicaçóes cardíacas graves como cardiomiopatia e insuficiência cardíaca congestiva ${ }^{43 ; 44}$. A cardiotoxicidade relacionada às antraciclinas cresce exponencialmente com a dose e pode ser irreversível ${ }^{44}$. A incidência de insuficiência cardíaca congestiva chega a 5\% dos casos, quando a dose cumulativa de doxorrubicina atinge $400 \mathrm{mg} / \mathrm{m}^{2}$. O risco de cardiotoxicidade é maior em mulheres idosas, hipertensas, com doença arterial coronariana preexistente e história previa de radioterapia mediastinal ${ }^{44}$. 
A existência de sobretratamento com quimioterapia ligado ao rastreamento mamográfico ainda não está bem estabelecida na literatura ${ }^{4,26}$. Em contrapartida, a redução do tratamento com quimioterapia - um possível benefício do rastreamento - também não apresenta comprovação científica adequada ${ }^{4,26}$. Em virtude do limitado efeito do rastreamento mamográfico na redução da incidência de casos avançados de câncer de mama, a redução de casos tratados com quimioterapia deve ser, no máximo, modesta e restrita a mulheres com mais de 50 anos, tendo em vista que o rastreamento de mulheres mais jovens não reduz a apresentação avançada da doença ${ }^{45,46}$.

Nos EUA, parece haver uma tendência recente de diminuição do uso de quimioterapia em pacientes com câncer de mama invasivo com receptor de estrogênio positivo, HER2 negativo e sem invasão de linfonodos ou com micrometástases, indo de 26,6\% (IC 95\%: 23,0\%$30,7 \%$ ) para $14,1 \%$ (IC 95\%: 12\%-16,3\% ${ }^{47}$. Essa queda, se mantida, certamente diminui a possibilidade de sobretratamento com quimioterapia.

Não se pretende aqui afirmar que não existe sobretratamento com quimioterapia de forma geral. Reconhece-se apenas que não há uma ligação estabelecida na literatura entre o rastreamento mamográfico e o aumento de tratamento com quimioterapia ${ }^{4}$; ou seja, não existe evidência conclusiva de sobretratamento com quimioterapia induzido pelo rastreamento mamográfico. Situação semelhante a esta ocorre com as evidências sobre hormonioterapia e terapia-alvo com trastuzumabe.

\section{SOBRETRATAMENTO COM TRASTUZUMABE}

$\mathrm{O}$ uso da terapia-alvo com o anticorpo monoclonal trastuzumabe, no subgrupo de mulheres com câncer de mama HER2 positivo, se difundiu na prática clínica a partir do final da década de 2010, passando a representar uma alternativa terapêutica tanto para casos de câncer de mama inicial quanto para metastáticos.

O principal risco associado a essa terapia é a cardiotoxicidade. Embora ela seja geralmente reversível com a descontinuidade do tratamento com trastuzumabe, alguns casos podem evoluir para insuficiência cardíaca grave e morte. Em uma série prospectiva e multicêntrica de 81 casos de câncer de mama inicial tratados com trastuzumabe no Brasil, $46 \%$ apresentaram fração de ejeção do ventrículo esquerdo menor do que 50\% ou queda de mais de $10 \%$ da fração de ejeção pré-tratamento e um caso evoluiu para óbito em virtude da cardiotoxicidade ${ }^{48}$. Há também relato da ocorrência de miocardipatia de Takotsubo após terapia com trastuzumabe e pertuzumabe ${ }^{49}$.

Como a terapia-alvo com trastuzumabe não era usada na época em que foram realizados os ensaios clínicos de rastreamento mamográfico, não há evidências conclusivas da existência de sobretratamento com trastuzumabe ou de sua redução com a prática do rastreamento. Portanto, não é possível afirmar que os riscos associados ao uso do trastuzumabe estejam aumentados ou reduzidos nas mulheres que se submetem ao rastreamento mamográfico.

\section{LIMITAÇÕES}

O presente estudo adotou a abordagem de revisão crítica da literatura, cuja ênfase está na contribuição conceitual de cada item da literatura incluída. Como outras revisóes dessa natureza, seu produto é uma hipótese praticamente ainda não explorada na literatura ${ }^{50}$, ligando o aumento de mortalidade cardiovascular associado ao diagnóstico e tratamento do câncer de mama, com o sobrediagnósrtico e sobretratamento oriundos do rastreamento mamográfico. A presente revisão possui todos os elementos das chamadas revisóes integrativas ${ }^{51}$. Embora tenha diversos aspectos típicos revisões sistemáticas da literatura, tais como perguntas de pesquisa, estratégias de busca e critérios de elegibilidade para seleção de artigos, outros foram omitidos, como busca da literatura cinzenta, seleção cega de títulos-resumos por mais de um avaliador e realização de metanálise. Essas escolhas deram-se também pela impossibilidade de se estudar diretamente os casos de sobrediagnóstico e sobretratamento, por sua natureza contrafactual, e pela necessidade de empreender diversas revisôes sobre cada um dos danos identificados. Além disso, não era pertinente realizar metanálise, uma vez que, para duas das estratégias de busca realizadas, a quantidade de estudos selecionados foi muito pequena e, para a radioterapia adjuvante, foi encontrada uma revisão sistemática com metanálise já realizada, optando-se então por uma apresentação narrativa dos resultados dos estudos. Portanto, como outros estudos classificados como revisóes críticas da literatura, a presente revisão se propóe a buscar uma interpretação nova para os dados existentes ${ }^{50}$. Dessa forma, pretendeu-se interpretar e correlacionar os achados aqui apresentados vis-à-vis ao corpo de evidências sobre danos do rastreamento mamográfico.

\section{CONCLUSÃO}

Embora a literatura sobre rastreamento mamográfico seja extensa, os danos cardiovasculares associados a essa prática têm sido sistematicamente ignorados. Existem evidências consistentes de aumento de mortalidade cardiovascular associada ao diagnóstico e ao tratamento do câncer de mama em situaçôes clinicamente compatíveis com o sobrediagnóstico e o sobretratamento derivados do rastreamento. É provável também que o aumento de mortalidade cardiovascular seja um dos danos mais 
importantes do rastreamento mamográfico, em especial o derivado da radioterapia adjuvante. Embora relativamente raros, os riscos cardiovasculares aqui abordados estáo sendo discutidos no contexto do sobrediagnóstico e do consequente sobretratamento, nos quais, por definição, não há benefícios que contrabalancem esses danos. A presente revisão não teve como objetivo comparar quantitativamente os danos cardiovasculares com o benefício do rastreamento mamográfico na reduçáo da mortalidade por câncer de mama, pois não há na literatura estudos que tenham quantificado, de forma abrangente, esse excesso de mortalidade e estimado o balanço entre riscos e benefícios. Esse é um tema ainda aberto que precisa ser mais bem explorado.

\section{CONTRIBUIÇÕES}

Arn Migowski concebeu o estudo, realizou a revisão de literatura, contribuiu na interpretação dos resultados, participou da redaçáo e da revisão do texto. Paulo Nadanovsky e Cid Manso de Mello Vianna contribuíram na interpretação dos resultados e com revisão crítica do artigo. Todos os autores leram e aprovaram a versão final do manuscrito e declaram ser responsáveis por todos os aspectos do trabalho, garantindo sua precisão e integridade.

\section{DECLARAÇÃO DE CONFLITO DE INTERESSES}

Nada a declarar.

\section{FONTES DE FINANCIAMENTO}

Não há.

\section{REFERÊNCIAS}

1. Migowski A, Stein AT, Ferreira CBT, et al. Guidelines for early detection of breast cancer in Brazil. I Development methods. Cad Saude Publica. 2018 Jun 21;34(6):e00116317. doi: http://dx.doi. org/10.1590/0102-311x00116317

2. Migowski A, Silva GA, Dias MBK, et al. Guidelines for early detection of breast cancer in Brazil. II - New national recommendations, main evidence, and controversies. Cad Saude Publica. 2018 Jun 21;34(6):e00074817. doi: http://dx.doi.org/10.1590/0102-311X00074817

3. Baines CJ, To T, Miller AB. Revised estimates of overdiagnosis from the Canadian National Breast Screening Study. Prev Med. 2016 Sep;90:66-71. doi: http://dx.doi.org/10.1016/j.ypmed.2016.06.033

4. Gotzsche PC, Jorgensen, KJ. Screening for breast cancer with mammography. Cochrane Database Syst
Rev. 2013 Jun 4;(6):CD001877. doi: http://dx.doi. org/10.1002/14651858.CD001877.pub5

5. Baum M. Harms from breast cancer screening outweigh benefits if death caused by treatment is included. BMJ. 2013 Jan 23;346:f385. doi: http://dx.doi.org/10.1136/ bmj.f385

6. Centre for Evidence-Based Medicine. OCEBM levels of evidence. [Internet] Oxford: CEBM; 2011. Table, The levels of evidence, version 2.1; [cited 2019 Jun 24]. Available from: https://www.cebm.net/wp-content/ uploads/2014/06/CEBM-Levels-of-Evidence-2.1.pdf

7. Fang F, Fall K, Mittleman MA, et al. Suicide and cardiovascular death after a cancer diagnosis. $\mathrm{N}$ Engl J Med. 2012 Apr 5;366(14):1310-1318. doi: http://dx.doi. org/10.1056/NEJMoa1110307

8. Ye Y, Otahal P, Marwick TH, et al. Cardiovascular and other competing causes of death among patients with cancer from 2006 to 2015: an Australian populationbased study. Cancer. 2019 Feb 1;125(3):442-452. doi: http://dx.doi.org/10.1002/cncr.31806

9. Henson KE, Reulen RC, Winter DL, et al. Cardiac mortality among 200000 five-year survivors of cancer diagnosed at 15 to 39 years of age: the teenage and young adult cancer survivor study. Circulation. $2016 \mathrm{Nov}$ 15;134(20):1519-1531. doi: http://dx.doi.org/10.1161/ CIRCULATIONAHA.116.022514

10. El-Tamer MB, Ward BM, Schifftner T, et al. Morbidity and mortality following breast cancer surgery in women: national benchmarks for standards of care. Ann Surg. 2007;245(5):665-671. doi: http://dx.doi. org/10.1097/01.sla.0000245833.48399.9a

11. Boekel NB, Schaapveld M, Gietema JA, et al. Cardiovascular morbidity and mortality after treatment for ductal carcinoma in situ of the breast. J Natl Cancer Inst. 2014 Aug 15;106(8):dju156. doi: http://dx.doi. org/10.1093/jnci/dju156

12. Taylor C, Correa C, Duane FK, et al. Estimating the risks of breast cancer radiotherapy: evidence from modern radiation doses to the lungs and heart and from previous randomized trials. J Clin Oncol. 2017;35(15):1641-1649. doi: http://dx.doi.org/10.1200/JCO.2016.72.0722

13. Sardar P, Kundu A, Chatterjee S, et al. Longterm cardiovascular mortality after radiotherapy for breast cancer: a systematic review and meta-analysis. Clin Cardiol. 2017;40(2):73-81. doi: http://dx.doi. org/10.1002/clc.22631

14. Demirci S, Nam J, Hubbs JL, et al. Radiation-induced cardiac toxicity after therapy for breast cancer: interaction between treatment era and follow-up duration. Int J Radiat Oncol Biol Phys. 2009 Mar 15;73(4):980-7. doi: http://dx.doi.org/10.1016/j.ijrobp.2008.11.016

15. Nolan MT, Russell DJ, Negishi K, et al. Meta-analysis of association between mediastinal radiotherapy and long-term heart failure. Am J Cardiol. 2016 Dec 
1;118(11):1685-1691. doi: http://dx.doi.org/10.1016/j. amjcard.2016.08.050

16. Gernaat SAM, Ho PJ, Rijnberg N, et al. Risk of death from cardiovascular disease following breast cancer: a systematic review. Breast Cancer Res Treat. 2017 Aug;164(3):537-555. doi: http://dx.doi.org/10.1007/ s10549-017-4282-9

17. Rozanski A, Blumenthal JA, Kaplan J. Impact of psychological factors on the pathogenesis of cardiovascular disease and implications for therapy. Circulation. 1999;99(16):2192-217. doi: http://dx.doi. org/10.1161/01.cir.99.16.2192

18. Rosengren A, Hawken S, Ounpuu S, et al. Association of psychosocial risk factors with risk of acute myocardial infarction in 11119 cases and 13648 controls from 52 countries (the INTERHEART study): case-control study. Lancet. 2004 Sep 11-17;364(9438):953-62. doi: https:// doi.org/10.1016/S0140-6736(04)17019-0

19. Vujcic I, Vlajinac H, Dubljanin E, et al. Psychosocial Stress and risk of myocardial infarction: a casecontrol study in Belgrade (Serbia). Acta Cardiol Sin. 2016;32(3):281-289. doi: https://doi.org/10.6515/ ACS20150424K

20. Li J, Hansen D, Mortensen PB, et al. Myocardial infarction in parents who lost a child: a nationwide prospective cohort study in Denmark. Circulation. 2002;106(13):1634-9. doi: https://doi.org/10.1161/01. CIR.0000031569.45667.58

21. Leor J, Poole WK, Kloner RA. Sudden cardiac death triggered by an earthquake. $\mathrm{N}$ Engl J Med. 1996;334(7):413-9. doi: https://doi.org/10.1056/ NEJM199602153340701

22. Kershaw KN, Brenes GA, Charles LE, et al. Associations of stressful life events and social strain with incident cardiovascular disease in the Women's Health Initiative. J Am Heart Assoc. 2014 Jun 27;3(3):e000687. doi: https:// doi.org/10.1161/JAHA.113.000687

23. Hatzaras IS, Bible JE, Koullias GJ, et al. Role of exertion or emotion as inciting events for acute aortic dissection. Am J Cardiol. 2007 Nov 1;100(9):1470-2. doi: https:// doi.org/10.1016/j.amjcard.2007.06.039

24. Konstantinos G, El-Battrawy I, Schramm K, et al. Comparison and outcome analysis of patients with takotsubo cardiomyopathy triggered by emotional stress or physical stress. Front Psychol. 2017;8:527. doi: https:// doi.org/10.3389/fpsyg.2017.00527

25. Dahabreh IJ, Wieland LS, Adam GP, et al. Core needle and open surgical biopsy for diagnosis of breast lesions: an update to the 2009 Report [Internet]. Rockville (MD): Agency for Healthcare Research and Quality (US); 2014 Sep (Comparative Effectiveness Reviews; no. 139). [cited 2019 June 26]. 248 p. Available from: https:/www.ncbi.nlm.nih.gov/books/NBK246878/pdf/ Bookshelf_NBK246878.pdf
26. Marmot MG, Altman DG, Cameron DA, et al. The benefits and harms of breast cancer screening: an independent review a report jointly commissioned by Cancer Research UK and the Department of Health (England). Br J Cancer. 2013 Jun 11;108(11):22052240. doi: https://doi.org/10.1038/bjc.2013.177

27. McGale P, Darby SC, Hall P, et al. Incidence of heart disease in 35,000 women treated with radiotherapy for breast cancer in Denmark and Sweden. Radiother Oncol. 2011 Aug;100(2):167-75. doi: https://doi.org/10.1016/j. radonc.2011.06.016

28. Burgy M, Brossat H, Barthelemy P, et al. First report of trastuzumab treatment after postoperative takotsubo cardiomyopathy. Anticancer Res. 2014 Jul;34(7):3579-82.

29. Boekel NB, Schaapveld M, Gietema JA, et al. Cardiovascular disease risk in a large, population-based cohort of breast cancer survivors. Int J Radiat Oncol Biol Phys. 2016;94(5):1061-72. doi: https://doi. org/10.1016/j.ijrobp.2015.11.040

30. Cheng YJ, Nie XY, Ji CC, et al. Long-Term Cardiovascular Risk After Radiotherapy in Women With Breast Cancer. J Am Heart Assoc. 2017;6(5):e005633. doi: https://doi. org/10.1161/JAHA.117.005633

31. Darby SC, Ewertz M, McGale P, et al. Risk of ischemic heart disease in women after radiotherapy for breast cancer. N Engl J Med. 2013 Mar 14;368(11):987-98. doi: https://doi.org/10.1056/NEJMoa1209825

32. Guy JB, Bertoletti L, Magné N, et al. Venous thromboembolism in radiation therapy cancer patients: Findings from the RIETE registry. Crit Rev Oncol Hematol. 2017 May;113:83-89. doi: https://doi. org/10.1016/j.critrevonc.2017.03.006

33. Clarke M, Collins R, Darby S, et al. Effects of radiotherapy and of differences in the extent of surgery for early breast cancer on local recurrence and 15-year survival: an overview of the randomised trials. Lancet. 2005 Dec 17;366(9503):2087-106. doi: https://doi. org/10.1016/S0140-6736(05)67887-7

34. Brown LC, Mutter RW, Halyard MY. Benefits, risks, and safety of external beam radiation therapy for breast cancer. Int J Womens Health. 2015 Apr 24;7:449-58. doi: https://doi.org/10.2147/IJWH.S55552

35. Sakka M, Kunzelmann L, Metzger M, et al. Cardiac dose-sparing effects of deep-inspiration breath-hold in left breast irradiation: is IMRT more beneficial than VMAT? Strahlenther Onkol. 2017 Oct;193(10):800811. doi: https://doi.org/10.1007/s00066-017-1167-0

36. Hickey BE, Lehman M, Francis DP, et al. Partial breast irradiation for early breast cancer. Cochrane Database Syst Rev. 2016 Jul 18;7:CD007077. doi: 10.1002/14651858.CD007077.pub3

37. Henson KE, McGale P, Taylor C, et al. Radiation-related mortality from heart disease and lung cancer more than 20 years after radiotherapy for breast cancer. Br J Cancer. 
2013;108(1):179-82. doi: https://doi.org/10.1038/ bjc. 2012.575

38. Kunkler IH, Williams LJ, Jack WJ, et al. Breastconserving surgery with or without irradiation in women aged 65 years or older with early breast cancer (PRIME II): a randomised controlled trial. Lancet Oncol. 2015 Mar;16(3):266-73. doi: https://doi.org/10.1016/S14702045(14)71221-5

39. Burstein HJ, Lacchetti C, Anderson H, et al. Adjuvant endocrine therapy for women with hormone receptorpositive breast cancer: American Society of Clinical Oncology Clinical Practice Guideline Update on ovarian suppression. J Clin Oncol. 2016;34(14):1689-701. doi: https://doi.org/10.1200/JCO.2015.65.9573

40. Haque R, Shi J, Schottinger JE, et al. Cardiovascular disease after aromatase inhibitor use. JAMA Oncol. 2016 Dec 1;2(12):1590-1597. doi: https://doi.org/10.1001/ jamaoncol.2016.0429

41. Ministério da Saúde (BR), Secretaria de Atenção à Saúde, Secretaria de Ciência, Tecnologia e Insumos Estratégicos. Portaria Conjunta no 04, de 23 de janeiro de 2018 [Internet]. [acesso 2019 jul. 02]. Disponível em: http://portalarquivos2.saude.gov.br/images/pdf/2018/ fevereiro/07/PORTARIA-no-04-PCDT.carcinoma. mama.2018.pdf

42. Zambetti M, Moliterni A, Materazzo C, et al. Long-term cardiac sequelae in operable breast cancer patients given adjuvant chemotherapy with or without doxorubicin and breast irradiation. J Clin Oncol. 2001;19(1):37-43. doi: https://doi.org/10.1200/JCO.2001.19.1.37

43. Colozza M, de Azambuja E, Cardoso F, et al. Breast cancer: achievements in adjuvant systemic therapies in the pre-genomic era. Oncologist. 2006 Feb;11(2):11125. doi: https://doi.org/10.1634/theoncologist.11-2-111

44. Azim HA Jr, de Azambuja E, Colozza M, et al. Long-term toxic effects of adjuvant chemotherapy in breast cancer. Ann Oncol. 2011 Sep;22(9):1939-47. doi: https://doi. org/10.1093/annonc/mdq683

45. Nelson HD, Cantor A, Humphrey L, et al. Screening for breast cancer: a systematic review to update the 2009 U.S. preventive services task force recommendation [Internet]. Rockville (MD): Agency for Healthcare Research and Quality (US); 2016 Jan. (Evidence Syntheses; no. 124). [cited 2019 June 28]. 286 p. Available from: https:// www.ncbi.nlm.nih.gov/books/NBK343819/pdf/ Bookshelf_NBK343819.pdf

46. Bleyer A, Welch HG. Effect of three decades of screening mammography on breast-cancer incidence. $\mathrm{N}$ Engl J Med. 2012 Nov 22;367(21):1998-2005. doi: https:// doi.org/10.1056/NEJMoa1206809

47. Kurian AW, Bondarenko I, Jagsi R, et al. Recent trends in chemotherapy use and oncologists' treatment recommendations for early-stage breast cancer. J Natl Cancer Inst. 2018;110(5):493-500. doi: https://doi. org/10.1093/jnci/djx239

48. Grazziotin LR, Picon PD. Observational study of trastuzumab-related cardiotoxicity in early and metastatic breast cancer. J Oncol Pharm Pract. 2017 Jun;23(4):264272. doi: https://doi.org/10.1177/1078155216639755

49. Lees C, Yazdan-Ashoori P, Jerzak KJ, et al. Takotsubo cardiomyopathy during anti-HER2 therapy for metastatic breast cancer. Oncologist. 2019;24(2):e80-e82. doi: https://doi.org/10.1634/theoncologist.2018-0285

50. Grant MJ, Booth A. A typology of reviews: an analysis of 14 review types and associated methodologies. Health Info Libr J. 2009 Jun;26(2):91-108. doi: https://doi. org/10.1111/j.1471-1842.2009.00848.x

51. Rocha SA, Bocchi SCM, Godoy MF. Acesso aos cuidados primários de saúde: revisão integrativa. Physis. 2016;26(1):87-111. doi: http://dx.doi.org/10.1590/ S0103-73312016000100007
Recebido em 5/7/2019. Aprovado em 17/9/2019. 